\title{
Uso de filmes de polietileno e absorção de etileno para o transporte refrigerado de maçã 'Gala'
}

\section{Use of polyethylene bags and ethylene absorption on refrigerated transport of 'Gala' apple fruit}

\author{
Auri Brackmann ${ }^{1 *}$; Ricardo Fabiano Hettwer Giehl²; Sérgio Tonetto de Freitas²; \\ Ana Cristina Eisermann ${ }^{3}$; Anderson Machado de Mello ${ }^{4}$
}

\section{Resumo}

Conduziu-se um experimento com o objetivo de avaliar o efeito do uso de filmes de polietileno (PE) de diferentes espessuras e o uso de sachês absorvedores de etileno sobre a composição gasosa presente nas embalagens e sobre a qualidade da maçã 'Gala', durante o período de transporte sob refrigeração. Os tratamentos foram obtidos pela combinação de duas espessuras de filmes de polietileno [polietileno de alta densidade (PEAD) de $7 \mathrm{~mm}$ e polietileno de baixa densidade (PEBD) de $20 \mathrm{~mm}$ ] e de diferentes quantidades de sachês absorvedores de etileno (permanganato de potássio) por embalagem $(0,1$ e 3 sachês). Cada unidade experimental constituiu-se de $18 \mathrm{~kg}$ de frutos, que haviam sido previamente armazenados a $0,5^{\circ} \mathrm{C}$ por um mês. Após este período, os frutos foram embalados nos filmes, acondicionados em caixas de papelão e submetidos durante 15 dias a $4^{\circ} \mathrm{C}$ e mais três dias a $20^{\circ} \mathrm{C}$. Verificou-se que o uso de filmes de PEBD de $20 \mu \mathrm{m}$ permitiu menores concentrações de $\mathrm{O}_{2}$ e de etileno e maiores de $\mathrm{CO}_{2}$, em relação ao filme de $7 \mu \mathrm{m}$, no interior das embalagens. Além disso, o uso de sachês absorvedores de etileno diminuiu a concentração de etileno. No entanto, devido ao curto período de armazenamento (15 dias) e à maturação dos frutos no momento da embalagem, a redução dos níveis de etileno não apresentou efeito significativo nos atributos qualitativos dos frutos.

Palavras-chave: Malus domestica Borkh, atmosfera modificada, polietileno de baixa densidade, polietileno de alta densidade

\footnotetext{
Abstract

The objective of this experiment was to evaluate the gaseous composition and quality maintenance of 'Gala' apples, stored within polyethylene (PE) bags of different thickness combined with ethylene absorption, during refrigerated transport. The treatments evaluated were combinations of two PE film thickness [high density polyethylene (HDPE) of $7 \mu \mathrm{m}$ and low density polyethylene (LDPE) of $20 \mu \mathrm{m}$ ] with different number of ethylene absorbents (potassium permanganate) per bag $(0,1$ and 3$)$. Each experimental unity contained $18 \mathrm{~kg}$ of fruits previously stored at $0.5^{\circ} \mathrm{C}$ during 1 month. After this period fruits were wrapped with the films and packed in cadboard boxes, being submitted during 15 days at $4^{\circ} \mathrm{C}$, simulating refrigerated transport plus 3 days of shelf-life at $20^{\circ} \mathrm{C}$. Lower levels of $\mathrm{O}_{2}$ and ethylene and higher of $\mathrm{CO}_{2}$ were observed within LDPE bags of $20 \mu \mathrm{m}$ than in HDPE bags of $7 \mu \mathrm{m}$. Moreover, the use of ethylene absorbents reduced the ethylene level within bags. However, due to the low storage time (15 days) and fruit ripeness at the packaging, the reduction of ethylene levels did not show significant effect on fruit quality.

Key words: Malus domestica Borkh, modified atmosphere, low density polyethylene, high density polyethylene

1 Engenheiro Agrônomo, Doutor em Ciências Agrárias, Professor Adjunto do Departamento de Fitotecnia da Universidade Federal de Santa Maria (UFSM), Santa Maria, RS. E-mail: brackman@ccr.ufsm.br.

2 Engenheiro Agrônomo, mestrando do Programa de Pós-graduação em Agronomia (PPGA) da UFSM. Bolsista do CNPq.

3 Acadêmica do curso de Agronomia da UFSM. Bolsista de Iniciação Científica do CNPq.

4 Engenheiro Agrônomo, Mestre em Agronomia, doutorando do PPGA da UFSM. Bolsista da CAPES.

* Autor para correspondêbcia 


\section{Introdução}

Para a manutenção da qualidade de maçãs, durante o transporte, é indispensável o uso de condições refrigeradas para diminuir a atividade respiratória. No caso da maçã 'Gala', para que o processo de armazenamento e transporte seja eficiente, deve-se considerar que os frutos dessa cultivar apresentam, em relação às demais cultivares, elevada taxa respiratória e alta produção de etileno, o que determina uma rápida perda de qualidade (BRACKMANN, 1992).

Para incrementar o efeito da baixa temperatura sobre a manutenção da qualidade dos frutos, podese fazer uso da atmosfera modificada (AM). Esta técnica permite a redução da taxa respiratória dos frutos, pois a combinação entre a respiração dos frutos e a permeabilidade do filme plástico aos gases provoca um aumento dos níveis de $\mathrm{CO}_{2}$ e uma diminuição das concentrações de $\mathrm{O}_{2}$ no interior da embalagem. Com isso, os processos metabólicos são modificados, pois a energia necessária para mantêlos é afetada (HERTOG; NICHOLSON; BANKS, 2001). Segundo Beaudry (1999), em filmes de polietileno de baixa densidade (PEBD) a permeabilidade ao $\mathrm{O}_{2}$ é aproximadamente $25 \%$ a do $\mathrm{CO}_{2}$. Knee (1980) investigou os efeitos de baixos níveis de $\mathrm{O}_{2}$ sobre a taxa de respiração e os processos de amadurecimento de maçãs 'Cox’s Orange Pippin' e concluiu que existe um paralelo evidente na conservação da qualidade e as concentrações de gases e umidade relativa obtidas com o uso de atmosfera modificada. Rocha, Barreira e Morais (2004), trabalhando com maçãs 'Bravo de Esmolfe', verificaram que os frutos armazenados em AM, durante 6,5 meses a $2^{\circ} \mathrm{C}$, apresentaram firmeza de polpa mais elevada em relação aos armazenados em ambiente refrigerado.

Em maçãs 'Gala', a absorção do etileno do ambiente de armazenamento permite a manutenção da firmeza de polpa, da acidez titulável e da cor de fundo da epiderme, além de diminuir a incidência de distúrbios fisiológicos (BRACKMANN et al., 2003).
No caso do armazenamento de frutos em embalagens, pode-se efetuar a eliminação do etileno mediante sua absorção através de sachês contendo um substrato impregnado com permanganato de potássio. Apesar de ser eficiente na manutenção da qualidade de maçãs 'Gala', durante o armazenamento, existem poucas informações a respeito da absorção do etileno em frutos embalados em filmes de polietileno.

O objetivo deste trabalho foi verificar o efeito do uso de filmes de polietileno (de alta e baixa densidade) de diferentes espessuras e o uso de sachês absorvedores de etileno sobre a composição gasosa presente no interior das embalagens e sobre a qualidade de maçãs 'Gala', após o transporte refrigerado.

\section{Material e Métodos}

O experimento foi conduzido no Núcleo de Pesquisa em Pós - colheita do Departamento de Fitotecnia da Universidade Federal de Santa Maria. Os frutos utilizados, da cv. Gala, foram adquiridos de um pomar comercial de Vacaria, RS, sendo submetidos a uma seleção, na qual foram excluídos os frutos defeituosos ou lesionados.

Os frutos foram armazenados durante um mês em ambiente refrigerado a $0,5^{\circ} \mathrm{C}$, antes de serem submetidos às condições de atmosfera modificada. Posteriormente, os frutos foram retirados da câmara frigorífica para serem embalados. Com isso, objetivou-se simular o procedimento adotado pelas empresas armazenadoras de maçãs, que, em algumas situações, efetuam a embalagem dos frutos apenas depois de determinado período de armazenamento, quando serão transportados para locais distantes.

Para a condução do experimento, o delineamento experimental utilizado foi o inteiramente casualizado, em esquema bifatorial, sendo cada tratamento constituído por quatro repetições. As unidades experimentais foram constituídas de cerca de $18 \mathrm{~kg}$ de frutos. Os tratamentos foram obtidos pela 
combinação de duas espessuras de filme de polietileno [polietileno de alta densidade (PEAD) de $7 \mathrm{~mm}$ e polietileno de baixa densidade (PEBD) de 20mm] e de diferentes quantidades de sachês absorvedores de etileno por embalagem ( 0,1 e 3$)$. Cada sachê absorvedor $\left(\right.$ Aceall $^{\circledR}$ ) consistia de cerca de $9 \mathrm{~g}$ de vermiculita impregnados com permanganato de potássio $\left(\mathrm{KMnO}_{4}\right)$.

As embalagens, contendo os frutos, foram acondicionadas em caixas de papelão corrugado e armazenadas durante 15 dias em uma câmara frigorífica a $4^{\circ} \mathrm{C}$, para simular as condições de transporte refrigerado. A temperatura da câmara frigorífica foi controlada por termostatos eletrônicos Full Gauge ${ }^{\circledR}$ (modelo MT-512Ri), sendo monitorada diariamente com o auxílio de termômetros de bulbo de mercúrio com $0,1^{\circ} \mathrm{C}$ de resolução, inseridos na polpa de um fruto.

Procedeu-se, a cada cinco dias de armazenamento, a leitura das concentrações de etileno, $\mathrm{CO}_{2}$ e $\mathrm{O}_{2}$ existentes no interior das embalagens. A concentração de etileno foi obtida pela injeção de amostras de $1 \mathrm{~mL}$ de cada embalagem em um cromatógrafo a gás marca Varian ${ }^{\circledR}$, modelo 3400CX, equipado com detector de ionização de chama (FID) e coluna de aço inox 1/8" de 0,70m de comprimento e preparada com Porapak N 80/100. Utilizou-se o $\mathrm{N}_{2}$ como gás de arraste a $30 \mathrm{~mL} \mathrm{~min}^{-1}$. As temperaturas da câmara de injeção, coluna e detector foram ajustadas em $90^{\circ} \mathrm{C}, 140^{\circ} \mathrm{C}$ e $200^{\circ} \mathrm{C}$, respectivamente. Já as concentrações de $\mathrm{O}_{2}$ e $\mathrm{CO}_{2}$, foram determinadas por meio da circulação da composição gasosa de cada embalagem em um analisador de gases, com fluxo contínuo, da marca Agri-Datalog ${ }^{\circledR}$ (modelo Oxidox II).

Depois de transcorridos 15 dias de armazenamento a $4^{\circ} \mathrm{C}$, os frutos foram retirados das embalagens e acondicionados numa câmara climatizada com temperatura de $20^{\circ} \mathrm{C}$, para simular as condições de comercialização. A análise laboratorial dos frutos foi realizada ao final de três dias a $20^{\circ} \mathrm{C}$. Os parâmetros avaliados foram: (a) cor de fundo da epiderme: determinada com o auxílio de um colorímetro da marca Minolta ${ }^{\circledR}$ (modelo CR-310), que utiliza o sistema CIELAB L*, $\mathrm{a}^{*}$, e b* de cores e os resultados expressos pela soma de $\mathrm{a}^{*}+\mathrm{b}^{*}$, sendo que quanto maior esse valor mais amarela e menos verde é a coloração de fundo da epiderme; (b) firmeza da polpa: avaliada na região equatorial dos frutos, em lados opostos, com um penetrômetro $\left(\right.$ Effegi $\left.^{\circledR}\right)$ manual com ponteira de $11,5 \mathrm{~mm}$ de diâmetro e os resultados expressos em Newton (N); (c) sólidos solúveis totais (SST): determinados com o auxílio de um refratômetro manual (Atago $\left.{ }^{\circledR}\right)$, com correção da temperatura e expressos em ${ }^{\circ}$ Brix; (d) acidez titulável: determinada através da titulação de uma solução, contendo $10 \mathrm{~mL}$ de suco diluído em $100 \mathrm{~mL}$ de água destilada, com $\mathrm{NaOH}(0,1 \mathrm{~N})$ até $\mathrm{pH} 8,1$, sendo os resultados expressos em meq $100 \mathrm{~mL}^{-1}$.

As médias para cada variável foram submetidas à análise da variância. As médias dos tratamentos foram comparadas ente si pelo teste de Duncan em nível de 5\% de probabilidade de erro.

\section{Resultados e Discussões}

De acordo com análise inicial, os frutos apresentavam firmeza da polpa de $69 \mathrm{~N}, 4,61 \mathrm{meq} 100 \mathrm{~mL}^{-1}$ de acidez titulável e $12,5^{\circ}$ Brix de sólidos solúveis totais. Após 15 dias a $4^{\circ} \mathrm{C}$ e mais três dias a $20^{\circ} \mathrm{C}$, não foi verificada interação significativa para os fatores espessura do filme de polietileno e número de sachês absorvedores de etileno por embalagem, para nenhum dos parâmetros avaliados (Tabelas 1, 2 e 3).

Menor concentração de $\mathrm{O}_{2}$ no interior das embalagens foi obtida com o uso do filme mais espesso (Tabela 1). As embalagens de PEBD de $20 \mu \mathrm{m}$ apresentavam $12,3 \%$ e $11,5 \%$ de $\mathrm{O}_{2}$ aos 10 e 15 dias de armazenamento refrigerado a $4{ }^{\circ} \mathrm{C}$, respectivamente (Tabela 1). Os níveis obtidos não foram suficientemente baixos para provocarem efeitos pronunciados na redução do metabolismo, pois segundo Beaudry (1999), níveis de $\mathrm{O}_{2}$ superiores a $5 \mathrm{kPa}$ não diminuem significativamente a atividade metabólica de tecidos vegetais. Nessas condições, a energia gerada ainda é suficiente para manter a maioria dos processos metabólicos primários e secundários, que ocorrem nas células dos frutos. 
Tabela 1. Concentrações de $\mathrm{O}_{2}$ e $\mathrm{CO}_{2}$ acumuladas no interior de embalagens de polietileno, contendo cerca de $18 \mathrm{~kg}$ de maçãs 'Gala' e acondicionadas em caixas de papelão corrugado, durante 15 dias a $4^{\circ} \mathrm{C}$. Santa Maria, RS.

\begin{tabular}{cccccc}
\hline Espessura do filme $(\mu \mathrm{m})$ & \multicolumn{3}{c}{$\mathrm{O}_{2}(\%)$} & \multicolumn{3}{c}{$\mathrm{CO}_{2}(\%)$} \\
\cline { 2 - 6 } & 10 dias & 15 dias & 5 dias $4{ }^{\circ} \mathrm{C}$ & 10 dias & 15 dias \\
\hline PEAD 7 & $17,2 \mathrm{a}^{2}$ & $18,4 \mathrm{a}$ & $3,4 \mathrm{~b}$ & $2,2 \mathrm{~b}$ & $1,89 \mathrm{~b}$ \\
PEBD 20 & $12,3 \mathrm{~b}$ & $11,5 \mathrm{~b}$ & $7,4 \mathrm{a}$ & $4,5 \mathrm{a}$ & $3,14 \mathrm{a}$ \\
$C V(\%)$ & 18,06 & 16,05 & 10,34 & 14,50 & 7,01 \\
\hline
\end{tabular}

${ }^{1} \mathrm{PEAD}=$ filme de polietileno de alta densidade; PEBD = filme de polietileno de baixa densidade.

${ }^{2}$ Médias seguidas de mesma letra, na vertical, não diferem entre si pelo teste de Duncan em nível de 5\% de probabilidade de erro.

A concentração de $\mathrm{CO}_{2}$ no interior das embalagens foi decrescente durante o período de armazenamento, independente da espessura do filme usado (Tabela 1). Provavelmente, as baixas concentrações de $\mathrm{O}_{2}$ e elevadas de $\mathrm{CO}_{2}$, no início do armazenamento, devem ter reduzido a taxa respiratória dos frutos. Com isso, o acúmulo de $\mathrm{CO}_{2}$ foi diminuindo, devido a sua menor produção e à permeabilidade do filme a esse gás. Com o uso de PEBD de 20 $\mu$ m obtiveram-se maiores concentrações de $\mathrm{CO}_{2}$ no interior das embalagens, durante o período avaliado (Tabela 1). Nessas embalagens a concentração de $\mathrm{CO}_{2}$ variou de 7,4\% a 3,1\% após cinco e 15 dias a $4^{\circ} \mathrm{C}$, respectivamente. Rocha, Barreira e Morais (2004), trabalhando com maçãs 'Bravo de Esmolfe', verificaram que, durante os primeiros 130 dias de armazenamento a $2^{\circ} \mathrm{C}$, as concentrações de $\mathrm{O}_{2}$ e $\mathrm{CO}_{2}$, no interior das embalagens de polipropileno de $100 \mu \mathrm{m}$, foram de $13,9 \%$ e 2,3\%, respectivamente. Após esse período, a concentração de $\mathrm{O}_{2}$ decresceu até $10 \%$ e a de $\mathrm{CO}_{2}$ aumentou até $5 \%$. O número de sachês absorvedores de etileno não afetou a concentração de $\mathrm{O}_{2}$ e $\mathrm{CO}_{2}$ no interior das embalagens (dados não apresentados).

Menor acúmulo de etileno foi obtido com o uso de sachês absorvedores de etileno (Tabela 2). Apesar das concentrações de etileno nas embalagens com um e três sachês absorvedores de etileno não terem diferenciado estatisticamente até os 10 dias de armazenamento, aos 15 dias a $4^{\circ} \mathrm{C}$ verificou-se menor concentração desse fito-hormônio com o uso de três sachês absorvedores de etileno (Tabela 2). No entanto, as concentrações de etileno obtidas foram ainda elevadas, pois a retenção da firmeza da polpa só foi conseguida com a manutenção de níveis inferiores a $0,4 \mu \mathrm{L} . \mathrm{L}^{-1}$ e $1,0 \mu \mathrm{L} . \mathrm{L}^{-1}$ para maçãs 'Gala' (BRACKMANN et al., 2003) e 'Empire' (BLANPIED, 1986), respectivamente. A espessura da embalagem também apresentou efeito no acúmulo de etileno no interior das embalagens (Tabela 2). $\mathrm{O}$ uso de PEBD de $20 \mu \mathrm{m}$ proporcionou menores concentrações de etileno a partir dos 10 dias de armazenamento, especialmente quando associado ao uso de sachês absorvedores de etileno. Possivelmente,

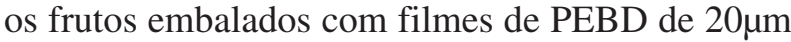
apresentaram menores taxas respiratórias, em virtude das menores concentrações de $\mathrm{O}_{2}$ e maiores de $\mathrm{CO}_{2}$ presentes no interior das embalagens (Tabela 1), pois, nessas condições, determinadas enzimas da rota glicolítica e do ciclo dos ácidos tricarboxílicos podem ter suas atividades reduzidas (MATHOOKO, 1996b). Além disso, as concentrações de $\mathrm{O}_{2}$ e $\mathrm{CO}_{2}$ podem ter reduzido a biossíntese de etileno, tanto pela menor disponibilidade de ATP (de WILD; WOLTERING; PEPPELENBOS, 1999), quanto pela inibição da ACC sintase e ACC oxidase pelo alto $\mathrm{CO}_{2}$ (MATHOOKO,1996a). 
Tabela 2. Concentração de etileno $\left(\mu \mathrm{L} \cdot \mathrm{L}^{-1}\right)$ no interior de embalagens de polietileno, contendo cerca de $18 \mathrm{~kg}$ de maçãs 'Gala' e acondicionadas em caixas de papelão corrugado, durante 15 dias a $4^{\circ} \mathrm{C}$. Santa Maria, RS.

\begin{tabular}{|c|c|c|c|c|c|c|c|c|c|}
\hline \multirow{2}{*}{$\begin{array}{l}\mathrm{N}^{\circ} \text { de sachês } \\
\text { absorvedores }\end{array}$} & \multicolumn{2}{|c|}{5 dias } & \multirow[t]{2}{*}{ Médias } & \multicolumn{2}{|c|}{10 dias } & \multirow[t]{2}{*}{ Médias } & \multicolumn{2}{|c|}{15 dias } & \multirow[t]{2}{*}{ Médias } \\
\hline & $\operatorname{PEAD} 7 \mu \mathrm{m}^{1}$ & PEBD $20 \mu \mathrm{m}$ & & PEAD $7 \mu \mathrm{m}$ & PEBD $20 \mu \mathrm{m}$ & & PEAD $7 \mu \mathrm{m}$ & PEBD $20 \mu \mathrm{m}$ & \\
\hline 0 & 52,74 & 85,99 & $69,36 \mathrm{a}^{2}$ & 77,96 & 33,82 & $55,89 \mathrm{a}$ & 98,88 & 51,31 & $75,10 \mathrm{a}$ \\
\hline 1 & 8,52 & 4,71 & $6,61 \mathrm{~b}$ & 20,46 & 0,95 & $10,71 \mathrm{~b}$ & 35,80 & 3,77 & $19,78 \mathrm{~b}$ \\
\hline 3 & 2,85 & 1,47 & $2,16 \mathrm{~b}$ & 5,46 & 0,86 & $3,16 \mathrm{~b}$ & 11,44 & 1,42 & $6,43 \mathrm{c}$ \\
\hline Médias & $21,37 \mathrm{~B}$ & $30,73 \mathrm{~A}$ & & $34,63 \mathrm{~A}$ & $11,88 \mathrm{~B}$ & & $48,70 \mathrm{~A}$ & $18,83 \mathrm{~B}$ & \\
\hline$C V(\%)$ & \multicolumn{2}{|c|}{14,52} & & \multicolumn{2}{|c|}{26,09} & \multicolumn{4}{|c|}{28,68} \\
\hline
\end{tabular}

${ }^{1} \mathrm{PEAD}=$ filme de polietileno de alta densidade $\mathrm{PEBD}=$ filme de polietileno de baixa densidade.

${ }^{2}$ Médias seguidas de mesma letra, minúscula na vertical e maiúscula na horizontal, não diferem entre si pelo teste de Duncan em nível de 5\% de probabilidade de erro.

Apesar da composição gasosa formada no interior das embalagens de polietileno ter sido diferente, em função de sua espessura e/ou do número de sachês absorvedores de etileno, os frutos não apresentaram diferença significativa na firmeza da polpa e nos teores de acidez e SST, após 15 dias $4^{\circ} \mathrm{C}$ e mais três dias a $20^{\circ} \mathrm{C}$ (Tabela 3 ). Houve pequena alteração nesses atributos, em relação à análise inicial, provavelmente em função do curto período de armazenamento e da maturação dos frutos no momento da embalagem. A redução dos níveis de etileno com o uso de sachês absorvedores de etileno e com os filmes de PEBD de $20 \mu$ m, não promoveu efeito significativo na retenção da firmeza da polpa dos frutos (Tabela 3). A esse respeito, Stow, Dover e Genge (2000) verificaram que baixas concentrações de etileno atrasam o início do processo de degradação da firmeza da polpa em maçãs ‘Cox’s Orange Pippin', mas não diminuem sua taxa quando já iniciado.

Tabela 3. Qualidade físico-química de maçãs 'Gala' embaladas em filmes de polietileno de diferentes espessuras e contendo sachês absorvedores de etileno, após 15 dias a $4^{\circ} \mathrm{C}$ e mais três dias a $20^{\circ} \mathrm{C}$. Santa Maria, RS.

\begin{tabular}{|c|c|c|c|c|c|c|c|c|c|}
\hline \multirow[t]{2}{*}{$\begin{array}{l}\mathrm{N}^{\mathrm{o}} \text { de sachês } \\
\text { absorvedores }\end{array}$} & \multicolumn{2}{|c|}{$\begin{array}{c}\text { Firmeza da polpa } \\
(\mathrm{N})\end{array}$} & \multirow[t]{2}{*}{ Médias } & \multicolumn{2}{|c|}{$\begin{array}{l}\text { Acidez titulável } \\
\left(\text { meq } 100 \mathrm{~mL}^{-1}\right)\end{array}$} & \multirow[t]{2}{*}{ Médias } & \multicolumn{2}{|c|}{$\begin{array}{l}\text { Cor de fundo da epiderme } \\
\qquad\left(a^{*}+b^{*}\right)\end{array}$} & \multirow[t]{2}{*}{ Médias } \\
\hline & $\overline{\mathrm{PEAD}} 7 \mu \mathrm{m}^{1}$ & PEBD $20 \mu \mathrm{m}$ & & PEAD $7 \mu \mathrm{m}$ & PEBD $20 \mu \mathrm{m}$ & & PEAD $7 \mu \mathrm{m}$ & PEBD $20 \mu \mathrm{m}$ & \\
\hline 0 & 68,9 & 69,0 & $68,9 \mathrm{a}^{2}$ & 4,48 & 4,62 & $4,55 \mathrm{a}$ & 49,97 & 45,99 & $47,98 \mathrm{a}$ \\
\hline 1 & 69,4 & 60,7 & $65,1 \mathrm{a}$ & 4,45 & 4,68 & $4,57 \mathrm{a}$ & 49,01 & 47,74 & $48,38 \mathrm{a}$ \\
\hline 3 & 67,5 & 67,2 & $67,4 \mathrm{a}$ & 4,44 & 4,52 & $4,48 \mathrm{a}$ & 49,70 & 48,62 & $49,16 \mathrm{a}$ \\
\hline Médias & $68,6 \mathrm{~A}$ & $65,6 \mathrm{~A}$ & & $4,46 \mathrm{~A}$ & $4,61 \mathrm{~A}$ & & $49,56 \mathrm{~A}$ & $47,45 \mathrm{~B}$ & \\
\hline$C V(\%)$ & \multicolumn{2}{|c|}{20,77} & & \multicolumn{2}{|c|}{4,82} & \multicolumn{4}{|c|}{3,39} \\
\hline
\end{tabular}

${ }^{1} \mathrm{PEAD}=$ filme polietileno de alta densidade PEBD = filme de polietileno de baixa densidade.

${ }^{2}$ Médias seguidas de mesma letra, minúscula na vertical e maiúscula na horizontal, não diferem entre si pelo teste de Duncan em nível de 5\% de probabilidade de erro.

Os frutos armazenados em embalagens de PEBD de $20 \mu \mathrm{m}$ estavam mais verdes, não ocorrendo diferença significativa para o número de sachês absorvedores de etileno por embalagem (Tabela 3). Stow e Genge (1990) e Stow, Dover e Genge (2000) não obtiveram efeito significativo sobre esse parâmetro com a remoção do etileno. Para Truter e Combrink (1993), o etileno aumenta a taxa de degradação das clorofilas somente após esse processo ter iniciado, sendo isso independente desse fitohormônio. A maior retenção da cor verde nos frutos armazenados em PEBD de $20 \mu \mathrm{m}$ pode ser explicada pelas menores concentrações de $\mathrm{O}_{2}$ obtidas com o uso dessa embalagem, pois Streif e Bangerth (1976) relataram efeito mais expressivo do $\mathrm{O}_{2}$ na degradação das clorofilas de tomate, em comparação ao etileno. 


\section{Conclusões}

Durante o transporte refrigerado de maçãs 'Gala' durante 15 dias, à temperatura de $4{ }^{\circ} \mathrm{C}$ e acondicionadas em caixas de papelão e embaladas em filmes plásticos, o uso de filmes de PEBD de $20 \mu \mathrm{m}$ reduz as concentrações de $\mathrm{O}_{2}$ e etileno e aumenta as de $\mathrm{CO}_{2}$. Além disso, o uso de sachês absorvedores de etileno no interior das embalagens reduziu significativamente a concentração de etileno. No entanto, no presente trabalho, os atributos qualitativos dos frutos praticamente não se alteraram independente da condição avaliada, provavelmente devido ao curto período de armazenamento e à maturação já avançada dos frutos no momento da embalagem. Dessa forma, o efeito da absorção do etileno não pôde ser devidamente avaliado.

\section{Referências}

BEAUDRY, R. M. Effect of $\mathrm{O}_{2}$ and $\mathrm{CO}_{2}$ partial pressure on selected phenomena affecting fruit and vegetable quality. Postharvest Biology and Technology, Amsterdam, v.15, p.293-303, 1999.

BLANPIED, G. D. A study of the relationship between fruit internal ethylene concentration and post-storage fruit quality of cv. Empire apples. The Journal of Horticultural Science, London, v.61, n.4, p.465-470, 1986.

BRACKMANN, A. Produção de etileno, $\mathrm{CO}_{2}$ e aroma de cultivares de maçã. Revista Brasileira de Fruticultura, Cruz das Almas, v.14, n.1, p.103-108, 1992.

BRACKMANN, A.; STEFFENS, C. A.; NEUWALD, D. A.; SESTARI, I. Armazenamento de maçã ' Gala' em atmosfera controlada com remoção de etileno. Ciência Rural, Santa Maria, v.33, n.4, p.647-650, 2003.

De WILD, H. P. J.; WOLTERING, E. J.; PEPPELENBOS, H. W. Carbon dioxide and 1-MCP inhibit ethylene production and respiration of pear fruit by different mechanisms. Journal of Experimental Botany, Oxford, v.50, n.335, p.837844, 1999.
HERTOG, M. L. A. T. M.; NICHOLSON, S. E.; BANKS, N. $\mathrm{H}$. The effect of modified atmospheres on the rate of firmness change in 'Braeburn' apples. Postharvest Biology and Technology, Amsterdam, v.23, n.3, p.175-184, 2001.

KNEE, M. Physiological responses of apple fruits to oxygen concentrations. Annals of Applied Biology, Warwick, v.96, n.2, p.243-253, 1980.

MATHOOKO, F. M. Regulation of ethylene biosynthesis in higher plants by carbon dioxide. Postharvest Biology and Technology, Amsterdam, v.7, n.1, p.1-26, 1996 .

MATHOOKO, F. M. Regulation of respiratory metabolism in fruits and vegetables by carbon dioxide. Postharvest Biology and Technology, Amsterdam, v.9, n.3, p.247-264, 1996b.

ROCHA, A. M. C. N.; BARREIRA, M. G.; MORAIS, A. M. M. B. Modified atmosphere package for apple 'Bravo de Esmolfe'. Food Control, Guildford, v.15, n.1, p.61-64, 2004.

STOW, J. R.; DOVER, C. J.; GENGE, P. M. Control of ethylene biosynthesis and softening in 'Cox's Orange Pippin' apples during low-ethylene, low-oxygen storage. Postharvest Biology and Technology, Amsterdam, v.18, n.3, p.215-225, 2000.

STOW, J. R.; GENGE, P. M. Low-ethylene storage of apples cv. 'Cox's Orange Pippin': effects of 'Rapid CA' and ultralow oxygen. Annals of Applied Biology, Warwick, v.117, n.3, p.673-681, 1990.

STREIF, J.; BANGERTH, F. The effect of differential partial pressures of oxygen and ethylene on the ripening of tomato fruits. Scientia Horticulturae, Amsterdam, v.5, p.227-237, 1976.

TRUTER, A. B.; COMBRINK, J. C. Ethylene levels in commercial CA and low-ethylene CA storage of Golden Delicious, Starking and Granny Smith apples and Packham's Triumph pears. Tree Fruit Postharvest Journal, Washington, v.4, n.3, p.14-18, 1993. 